\title{
3D Spatial Fading Correlation for Uniform Angle of Arrival Distribution
}

\author{
Yibeltal F. Alem, Student Member, IEEE, Zubair Khalid, Member, IEEE and Rodney A. Kennedy, Fellow, IEEE
}

\begin{abstract}
We derive a closed-form expression for the spatial fading correlation (SFC) between two arbitrary points in 3Dspace for the uniform limited azimuth-elevation angle of arrival probability density function (pdf). This expression simplifies the computation of the SFC, can be used in any 3D antenna array geometry, and avoids the need to generate separate expressions for specific antenna array geometries. We corroborate the accuracy of the closed-form expression through application to 2D and 3D antenna array geometries. We expect the results presented in this letter to be of significant importance for performance evaluation and sensitivity analysis in multi-input multi-output (MIMO) systems.
\end{abstract}

Index Terms-Spatial correlation, antenna arrays, MIMO, uniform distribution, spherical harmonics.

\section{INTRODUCTION}

In multi-input multi-output (MIMO) systems, the accurate characterisation of the spatial correlation of fading signals between the antenna array elements is of significant importance in determining the capacity and signal quality of such systems [1]-[3]. In this work, we consider the problem of accurately determining the spatial fading correlation (SFC) function of a multiple antenna array for the distribution of angle-of-arrival (AoA) that is uniformly distributed over a spatial region of limited azimuth and elevation. This is referred to as the "uniform limited azimuth-elevation AoA probability density function (pdf)" or simply the "uniform AoA pdf".

The SFC for several 2D AoA spatial distributions, which take into account only the azimuth of an incident signal, and 3D AoA spatial distributions, which take into account both azimuth and elevation, have been extensively studied and various closed-form expressions have been given [1], [4]-[9]. In particular, the uniform AoA pdf has been used extensively to model the distribution of scatterers and compute the spatial correlation experienced between multiple-antenna elements to evaluate the performance of MIMO systems [1], [4], [8], [10][14].

For the uniform AoA pdf, the closed-form SFC function has been determined for uniform linear array (ULA), uniform circular array (UCA), uniform rectangular array (URA) and electromagnetic vector sensors (EVS) antenna arrays [1]. Using an alternative approach that uses the spherical harmonic expansion of plane-waves, an expression for the SFC for the same uniform AoA pdf was given in [6]. Although

The authors are with Research School of Engineering, The Australian National University, Canberra, ACT 2601, Australia. Email: \{yibeltal.alem,zubair.khalid,rodney.kennedy\}@anu.edu.au. This work was supported by the Australian Research Council's Discovery Projects funding scheme (project no. DP150101011). the methodology used in [6] has the advantage that it is independent of the geometry of antenna array, the expression given for the uniform AoA pdf SFC was not in closedform nor in the most general form. Using the methodology from [6], this work finds such a closed-form SFC expression for the uniform AoA pdf. In comparison to the rotationally symmetric distributions for which closed-form SFC function has been presented in the literature, we note that the uniform distribution, being considered here, offers flexibility to model directional AoA distribution. The proposed SFC expression enables an exact computation of spatial correlation and is expected to be of great use for the performance evaluation of MIMO systems [1], [8], [12].

We first review the necessary mathematical background in Section II and then we derive the closed-form expression for SFC in Section III, where we also numerically validate the accuracy of the closed-form SFC for uniform linear, uniform circular and regular dodecahedron antenna array geometries.

\section{Spatial CORRElation - BACKGRound}

In MIMO systems, the 3D multipath channel impulse response for a signal arriving at antenna array is characterized by the steering vector of the antenna array. For an antenna array consisting of $M$ antenna elements placed at $\boldsymbol{z}_{p} \in \mathbb{R}^{3}, p=$ $1,2, \ldots, M$, the steering vector, denoted by $\boldsymbol{\alpha}(\widehat{\boldsymbol{x}})$, is given by

$$
\boldsymbol{\alpha}(\widehat{\boldsymbol{x}})=\left[\alpha_{1}(\widehat{\boldsymbol{x}}), \alpha_{2}(\widehat{\boldsymbol{x}}), \ldots \alpha_{M}(\widehat{\boldsymbol{x}})\right], \quad \alpha_{p}(\widehat{\boldsymbol{x}}) \triangleq e^{i k \boldsymbol{z}_{p} \cdot \widehat{\boldsymbol{x}}}
$$

where $\widehat{\boldsymbol{x}} \in \mathbb{R}^{3}$ denotes a unit vector pointing in the direction of wave propagation and $k=2 \pi / \lambda$ with $\lambda$ denoting the wavelength of the arriving signal. Since the unit vector $\widehat{\boldsymbol{x}} \in \mathbb{R}^{3}$ represents a point on the unit sphere, denoted by $\mathbb{S}^{2} \subset \mathbb{R}^{3}$, we also use its representation in spherical coordinates, that is, $\widehat{\boldsymbol{x}}=\widehat{\boldsymbol{x}}(\theta, \phi) \triangleq(\sin \theta \cos \phi, \sin \theta \sin \phi, \cos \theta)^{\prime} \in \mathbb{S}^{2} \subset \mathbb{R}^{3}$, where $(\cdot)^{\prime}$ denotes vector transpose, $\theta \in[0, \pi]$ denotes the elevation (strictly the co-latitude since $\theta=0$ is the zenith) and $\phi \in[0,2 \pi)$ denotes the azimuth (longitude).

\section{A. Functions on the Unit Sphere}

Spherical harmonics serve as orthonormal basis functions for the representation of functions on the sphere and are defined for integer degree $\ell \geq 0$ and integer order $|m| \leq \ell$ as

$$
Y_{\ell}^{m}(\widehat{\boldsymbol{x}}) \equiv Y_{\ell}^{m}(\theta, \phi) \triangleq \sqrt{\frac{2 \ell+1}{4 \pi} \frac{(\ell-m) !}{(\ell+m) !}} P_{\ell}^{m}(\cos \theta) e^{i m \phi},
$$

where $P_{\ell}^{m}(\cdot)$ denotes the associated Legendre polynomial of degree $\ell$ and order $m$ [15]. Any finite energy function $f(\widehat{\boldsymbol{y}})$ 
defined on the unit sphere, where $\widehat{\boldsymbol{y}} \in \mathbb{S}^{2} \subset \mathbb{R}^{3}$, can be expanded as

$$
f(\widehat{\boldsymbol{y}})=\sum_{\ell=0}^{\infty} \sum_{m=-\ell}^{\ell}\left(\int_{\mathbb{S}^{2}} f(\widehat{\boldsymbol{x}}) \overline{Y_{\ell}^{m}(\widehat{\boldsymbol{x}})} d s(\widehat{\boldsymbol{x}})\right) Y_{\ell}^{m}(\widehat{\boldsymbol{y}}) .
$$

Here $\overline{(\cdot)}$ denotes the complex conjugate operation and $d s(\widehat{\boldsymbol{x}})=$ $\sin \theta d \theta d \phi$.

\section{B. Spatial Fading Correlation}

Let $f(\widehat{x})$ denote the pdf of the angles of arrival of the multipath components or the unit-normalized power of a signal received from the direction $\widehat{x}$. Then the SFC function between $p$-th and $q$-th antenna elements, located at $\boldsymbol{z}_{p}$ and $\boldsymbol{z}_{q}$, respectively, with an assumption that signals arriving at the antenna elements are narrowband, is given by [1], [6], [7]

$$
\begin{aligned}
\rho\left(\boldsymbol{z}_{p}, \boldsymbol{z}_{q}\right) & \triangleq \int_{\mathbb{S}^{2}} f(\widehat{\boldsymbol{x}}) \alpha_{p}(\widehat{\boldsymbol{x}}) \overline{\alpha_{q}(\widehat{\boldsymbol{x}})} d s(\widehat{\boldsymbol{x}}) \\
& =\int_{\mathbb{S}^{2}} f(\widehat{\boldsymbol{x}}) e^{i k\left(\boldsymbol{z}_{p}-\boldsymbol{z}_{q}\right) \cdot \widehat{\boldsymbol{x}}} d s(\widehat{\boldsymbol{x}}) \equiv \rho\left(\boldsymbol{z}_{p}-\boldsymbol{z}_{q}\right),
\end{aligned}
$$

which indicates that the SFC only depends on $\boldsymbol{z}_{p}-\boldsymbol{z}_{q}$ and is, therefore, spatially wide-sense stationary.

\section{3D SPATIAL CORRELATION FOR UNIFORM DISTRIBUTION}

First we define a limited azimuth-elevation region [6]

$R=\left\{(\theta, \phi): \theta \in\left[\theta_{\circ}-\Delta_{\theta}, \theta_{\circ}+\Delta_{\theta}\right], \phi \in\left[\phi_{\circ}-\Delta_{\phi}, \phi_{\circ}+\Delta_{\phi}\right]\right\}$,

which is the Cartesian product of a range of positive elevations and limited azimuths. Here, $\theta_{\circ}$ and $\phi_{\circ}$ denote the mean elevation of arrival (MEOA) and the mean azimuth of arrival (MAOA), respectively, and $\Delta_{\theta}$ and $\Delta_{\phi}$ represent elevation spread (ES) and azimuth spread (AS).

Definition 1 (Uniform limited azimuth-elevation AoA pdf).

$$
f(\widehat{\boldsymbol{x}})= \begin{cases}\frac{1}{C\left(\theta_{\circ}, \Delta_{\theta}, \Delta_{\phi}\right)}, & \widehat{\boldsymbol{x}} \in R, \\ 0, & \widehat{\boldsymbol{x}} \in \mathbb{S}^{2} \backslash R,\end{cases}
$$

where $C\left(\theta_{\circ}, \Delta_{\theta}, \Delta_{\phi}\right)=\int_{R} d s(\widehat{\boldsymbol{x}})=4 \Delta_{\phi} \sin \theta_{\circ} \sin \Delta_{\theta}$. The pdf satisfies $\int_{\mathbb{S}^{2}} f(\widehat{\boldsymbol{x}}) d s(\widehat{\boldsymbol{x}})=1$.

As motivated in the introduction, in the following theorem, we find a closed-form expression for the SFC function, (3), when $f(\widehat{x})$ is the uniform AoA pdf.

Theorem 1. [Spatial fading correlation for uniform AoA pdf] If the AoA of the signals received at two antenna elements placed at $\boldsymbol{z}_{p}, \boldsymbol{z}_{q} \in \mathbb{R}^{3}$ follow the uniform AoA pdf (4), then the SFC, (3), between the antenna elements is given by

$$
\begin{aligned}
& \rho\left(\boldsymbol{z}_{p}-\boldsymbol{z}_{q}\right)= \frac{i^{m} 2 \sqrt{\pi}}{C\left(\theta_{\circ}, \Delta_{\theta}, \Delta_{\phi}\right)} \sum_{\ell=0}^{\infty} i^{\ell} j_{\ell}\left(k\left\|\boldsymbol{z}_{p}-\boldsymbol{z}_{q}\right\|\right) \times \\
& \sum_{m=-\ell}^{\ell} \sqrt{2 \ell+1} Q\left(m ; \phi_{\circ}, \Delta_{\phi}\right) \times \\
& \sum_{u=-\ell}^{\ell} d_{u, m}^{\ell}\left(\frac{\pi}{2}\right) d_{u, 0}^{\ell}\left(\frac{\pi}{2}\right) S\left(u ; \theta_{\circ}, \Delta_{\theta}\right) Y_{\ell}^{m}\left(\frac{\boldsymbol{z}_{p}-\boldsymbol{z}_{q}}{\left\|\boldsymbol{z}_{p}-\boldsymbol{z}_{q}\right\|}\right),
\end{aligned}
$$

where $j_{\ell}(\cdot)$ denotes the spherical Bessel function of first kind, $d_{u, m}^{\ell}(\cdot)$ denotes the Wigner-d function of degree $\ell$ and orders $u, m$ [15],

$$
Q\left(m ; \phi_{\circ}, \Delta_{\phi}\right)=2 e^{i m \phi_{\circ}} \frac{\sin \left(m \Delta_{\phi}\right)}{m},
$$

and

$$
S\left( \pm 1 ; \theta_{\circ}, \Delta_{\theta}\right)= \pm i\left(\Delta_{\theta}-\frac{1}{2} e^{ \pm 2 i \theta_{\circ}}\right)
$$

for $u= \pm 1$, and

$$
\begin{aligned}
& S\left(u ; \theta_{\circ}, \Delta_{\theta}\right)= \\
& \frac{e^{i u\left(\theta_{\circ}-\Delta_{\theta}\right)}\left(-\cos \left(\theta_{\circ}-\Delta_{\theta}\right)+i u \sin \left(\theta_{\circ}-\Delta_{\theta}\right)\right)}{u^{2}-1} \\
& +\frac{e^{i u\left(\theta_{\circ}+\Delta_{\theta}\right)}\left(\cos \left(\theta_{\circ}+\Delta_{\theta}\right)-i u \sin \left(\theta_{\circ}+\Delta_{\theta}\right)\right)}{u^{2}-1},
\end{aligned}
$$

whenever $u \neq \pm 1$.

Proof. See Appendix A.

Remark 1. It is noted that the uniform limited azimuthelevation AoA distribution is directional in nature and is, therefore, capable to model directional characteristics of AoA distribution. To the best of our knowledge, the closed-form SFC function that only depends on the location of antennas has only been derived for non-directional rotationally symmetric distributions (e.g., [6], [7], [9]).

Remark 2. If the multipath components arise from clusters of scatterers [16], the AoA of the signal is modelled by a mixture (positive weighted sum) of uniform limited azimuthelevation distributions, each with a different set of parameters. For such mixture distribution with pdf $g(\widehat{\boldsymbol{x}})$ defined as

$$
g(\widehat{\boldsymbol{x}}) \triangleq \sum_{w=1}^{W} \alpha_{w} f_{w}(\widehat{\boldsymbol{x}}), \quad \text { with } \alpha_{w} \in \mathbb{R}^{+}, \sum_{w=1}^{W} \alpha_{w}=1
$$

the SFC between two antenna elements placed at $\boldsymbol{z}_{p}, \boldsymbol{z}_{q} \in \mathbb{R}^{3}$, due to the linearity of the integral in (3) or (9), is given by a weighted sum of SFC function $\rho\left(\boldsymbol{z}_{p}-\boldsymbol{z}_{q}\right)$ derived in Theorem 1 for each of the uniform limited azimuth-elevation distribution in the mixture.

\section{A. Computation of Spatial Fading Correlation}

In the computation of SFC using the proposed formulation, given in (5), the summation for $\ell$ over first few terms yields sufficient accuracy as higher order Bessel functions decay rapidly to zero for points near each other in space as noted in [6], [17]. For the computation of SFC, we are required to compute Wigner- $d$ functions $d_{u, m}^{\ell}(\pi / 2)$ for each $|u|,|m| \leq \ell$. Let $\mathbf{D}_{\ell}$ denote the matrix of size $(2 \ell+1) \times(2 \ell+1)$ with entries $d_{u, m}^{\ell}(\pi / 2)$ for $|u|,|m| \leq \ell$. The matrix $\mathbf{D}_{\ell}$ can be computed for each $\ell=1,2, \ldots$, using the relation given in [18] that recursively computes $\mathbf{D}_{\ell}$ from $\mathbf{D}_{\ell-1}$. 


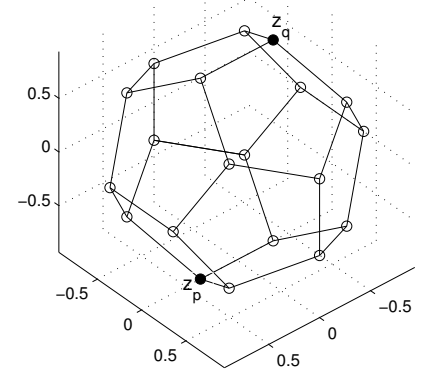

Fig. 1. RDA of 20 antenna elements placed at the vertices of regular dodecahedron inscribed in a sphere of radius $R=1$. Antenna elements at $\boldsymbol{z}_{p}=R \times(0.3568,0,-0.9342)^{\prime}$ and $\boldsymbol{z}_{q}=R \times(-0.3568,0,0.9342)^{\prime}$ are shaded.

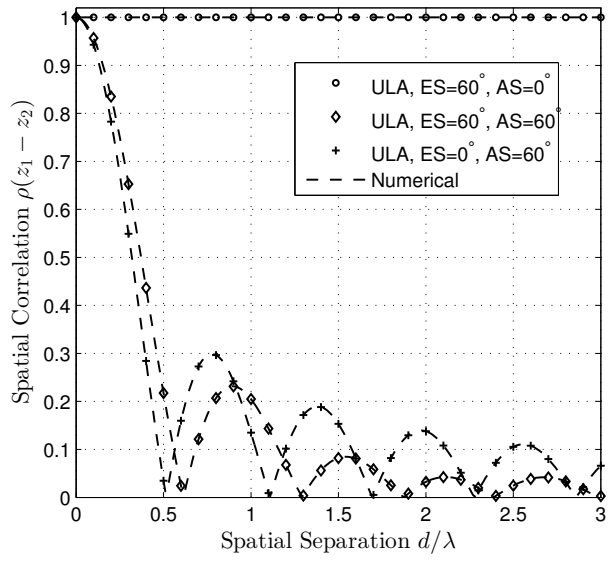

Fig. 2. Magnitude of the SFC function $\rho\left(\boldsymbol{z}_{1}-\boldsymbol{z}_{2}\right)$ between ULA antenna elements 1 and 2 spatially separated by distance $d$. MEOA=MAOA $=90^{\circ}$.

\section{B. Numerical Validation}

Here, we validate the proposed result through numerical experiments. We consider ULA, UCA (for 2D case) and regular dodecahedron array (RDA for 3D case) in our analysis. The antenna elements of a ULA are placed at the following spatial positions: $\left(\boldsymbol{z}_{p}\right)_{\mathrm{ULA}}=(0+d(p-1), 0,0)^{\prime} \in \mathbb{R}^{3}$, where $d$ denotes the separation distance between the ULA antenna elements. Similarly, the antenna elements of an $M$ element UCA are placed at the following spatial positions: $\left(\boldsymbol{z}_{p}\right)_{\mathrm{UCA}}=\left(R \cos \frac{2 \pi p}{M}, R \sin \frac{2 \pi p}{M}, 0\right)^{\prime} \in \mathbb{R}^{3}$, where $R$ denotes the radius of the array for UCA. For the RDA, the 20 antenna elements are placed at the vertices of dodecahedron inscribed in a sphere of radius $R$ as shown in Fig. 1 (for $R=1$ ). Using the proposed closed-form expression for the SFC function, we determine $\rho\left(\boldsymbol{z}_{1}-\boldsymbol{z}_{2}\right)$ for ULA, $\rho\left(\boldsymbol{z}_{1}-\boldsymbol{z}_{2}\right)$ for 4 element UCA and $\rho\left(\boldsymbol{z}_{p}-\boldsymbol{z}_{q}\right)$ for RDA, where $\boldsymbol{z}_{p}$ and $z_{q}$ are indicated in Fig. 1. For different uniform limited azimuth-elevation distributions of AoA, we plot the magnitude of the SFC function against $R / \lambda$ for ULA, UCA and RDA in Fig. 2, Fig. 3 and Fig. 4, respectively, where we also plot the numerically evaluated SFC, obtained using (3), which matches with the proposed SFC function and thus corroborates the correctness of proposed SFC function.

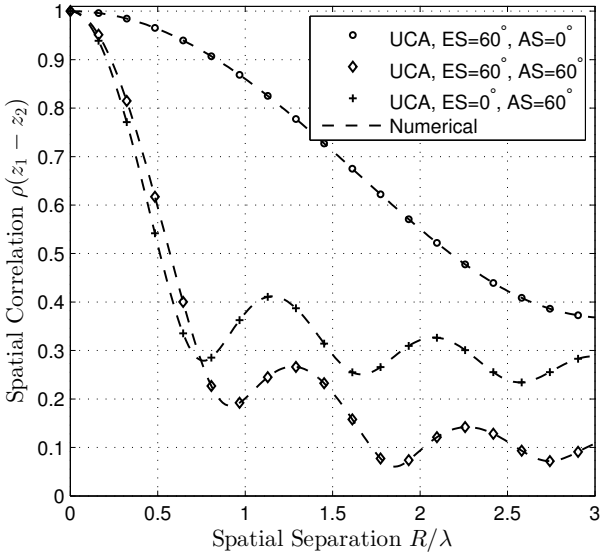

Fig. 3. Magnitude of the SFC function $\rho\left(\boldsymbol{z}_{1}-\boldsymbol{z}_{2}\right)$ for 4 element UCA of radius $R$. MEOA $=\mathrm{MAOA}=90^{\circ}$.

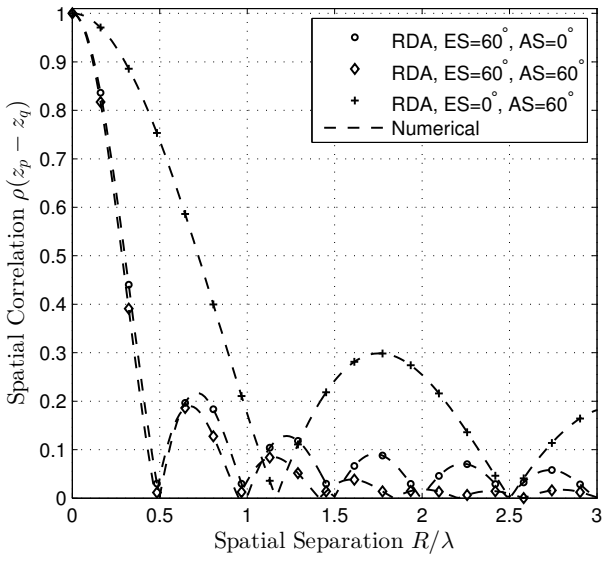

Fig. 4. Magnitude of the SFC function $\rho\left(\boldsymbol{z}_{p}-\boldsymbol{z}_{q}\right)$ for RDA of radius $R$ with MEOA $=\mathrm{MAOA}=90^{\circ} . \boldsymbol{z}_{p}$ and $\boldsymbol{z}_{q}$ are indicated in Fig. 1.

\section{Impact of Angular Spread on Array Response}

We compare the impact of change in azimuth of arrival (AOA) and elevation of arrival (EOA) on MIMO system performance. We employ the proposed closed-form expression to perform the comparison by computing the SFC between two antenna elements using UCA (2D) and RDA (3D). We plot the change in the magnitude of the SFC with respect to change in ES and AS at $0.5 \lambda$ spacing, $90^{\circ} \mathrm{MAOA}$ and MEOA as illustrated in Fig. 5 and Fig. 6 for 2D array and 3D array, respectively. It can be observed that, in the $2 \mathrm{D}$ array case, an increase in AS has more significant impact on the magnitude of the SFC than an increase in ES. However, in the 3D array case it is noted that the magnitude of the SFC is significantly affected by an increase in both AS and ES, suggesting that the 3D antenna array geometries offer more flexibility in achieving lower spatial correlation and consequently improving system performance as also reported in [8].

\section{CONClusions}

For the uniform limited azimuth-elevation AoA pdf, we have derived a closed-form expression for SFC between two arbitrary points in 3D-space and, hence, can be applied to any antenna array geometry. This simplifies the computation of the SFC and avoids the need to generate separate expressions for 


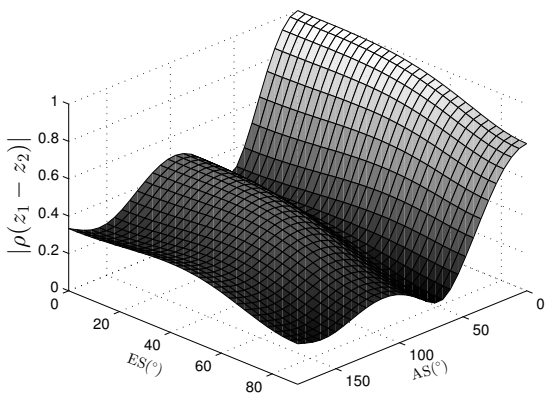

Fig. 5. Magnitude of the SFC as a function of both AS and ES for UCA (2D geometry).

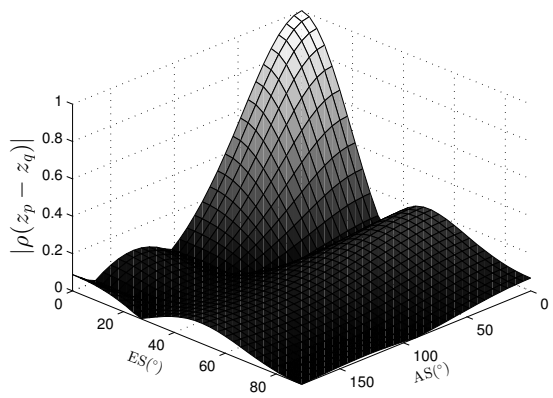

Fig. 6. Magnitude of the SFC as a function of both AS and ES for RDA (3D geometry).

specific array geometries. Furthermore, we have shown that the proposed closed-form expression is useful for performing sensitivity analysis of antenna arrays.

\section{APPENDIX A - PROOF OF THEOREM 1}

Using spherical harmonic expansion of plane waves [19]:

$$
e^{i k \boldsymbol{z}_{p} \cdot \widehat{\boldsymbol{x}}}=4 \pi \sum_{\ell=0}^{\infty} i^{\ell} j_{\ell}\left(k\left\|\boldsymbol{z}_{p}\right\|\right) \sum_{m=-\ell}^{\ell} Y_{\ell}^{m}\left(\boldsymbol{z}_{p} /\left\|\boldsymbol{z}_{p}\right\|\right) \overline{Y_{\ell}^{m}(\widehat{\boldsymbol{x}})},
$$

and (2) and employing the orthonormality of spherical harmonics, we first write the SFC function in (3) as [6]

$$
\begin{aligned}
\rho\left(\boldsymbol{z}_{p}-\boldsymbol{z}_{q}\right) & =\sum_{\ell=0}^{\infty} i^{\ell} j_{\ell}\left(k\left\|\boldsymbol{z}_{p}-\boldsymbol{z}_{q}\right\|\right) \sum_{m=-\ell}^{\ell} Y_{\ell}^{m}\left(\frac{\boldsymbol{z}_{p}-\boldsymbol{z}_{q}}{\left\|\boldsymbol{z}_{p}-\boldsymbol{z}_{q}\right\|}\right) \\
& \times \frac{4 \pi}{C\left(\theta_{\circ}, \Delta_{\theta}, \Delta_{\phi}\right)} \int_{R} Y_{\ell}^{m}(\widehat{\boldsymbol{x}}) d s(\widehat{\boldsymbol{x}}),
\end{aligned}
$$

where the integral, over the region $R$, can be decomposed as

$$
\begin{aligned}
\int_{R} Y_{\ell}^{m}(\widehat{\boldsymbol{x}}) d s(\widehat{\boldsymbol{x}})=\sqrt{\frac{2 \ell+1}{4 \pi} \underbrace{\int_{\phi_{\circ}-\Delta_{\phi}}^{\phi_{\circ}+\Delta_{\phi}} e^{i m \phi} d \phi}_{Q\left(m ; \phi_{\circ}, \Delta_{\phi}\right)}} \\
\times \underbrace{\sqrt{\frac{(\ell-m) !}{(\ell+m) !} \int_{\theta_{\circ}-\Delta_{\theta}}^{\theta_{\circ}+\Delta_{\theta}} P_{\ell}^{m}(\cos \theta) \sin \theta d \theta}}_{G\left(u ; \theta_{\circ}, \Delta_{\theta}\right)} .
\end{aligned}
$$

An expression for $Q\left(m ; \phi_{\circ}, \Delta_{\phi}\right)$ is given in (6). To determine $G\left(u ; \theta_{\circ}, \Delta_{\theta}\right)$, we use the following relation between the associated Legendre function and the Wigner- $d$ function [15]:

$$
P_{\ell}^{m}(\cos \theta)=\sqrt{\frac{(\ell+m) !}{(\ell-m) !}} d_{\ell}^{m, 0}(\theta),
$$

and the following expansion of Wigner- $d$ functions in terms of complex exponentials [15], [20]

$$
d_{m, n}^{\ell}(\theta)=i^{n-m} \sum_{u=-\ell}^{\ell} d_{u, m}^{\ell}(\pi / 2) d_{u, n}^{\ell}(\pi / 2) e^{i u \theta} .
$$

By defining

$$
S\left(u ; \theta_{\circ}, \Delta_{\theta}\right) \triangleq \int_{\theta_{\circ}-\Delta_{\theta}}^{\theta_{\circ}+\Delta_{\theta}} e^{i u \theta} \sin \theta d \theta
$$

which is evaluated in (7b), and substituting backwards from (12) to (10) in (9) yields the SFC function given in (5).

\section{REFERENCES}

[1] S. K. Yong and J. Thompson, "Three-dimensional spatial fading correlation models for compact MIMO receivers," IEEE Trans. Wireless Commun., vol. 4, no. 6, pp. 2856-2869, Nov. 2005.

[2] R. A. Kennedy, P. Sadeghi, T. D. Abhayapala, and H. M. Jones, "Intrinsic limits of dimensionality and richness in random multipath fields," IEEE Trans. Signal Process., vol. 55, no. 6, pp. 2542-2556, Jun. 2007.

[3] D. S. Shiu, G. J. Foschini, M. J. Gans, and J. M. Kahn, "Fading correlation and its effect on the capacity of multielement antenna systems," IEEE Trans. Commun., vol. 48, no. 3, pp. 502-513, Mar. 2000.

[4] J. Salz and J. H. Winters, "Effect of fading correlation on adaptive arrays in digital mobile radio," IEEE Trans. Veh. Technol., vol. 43, no. 4, pp. 1049-1057, Nov. 1994.

[5] J.-A. Tsai, R. M. Buehrer, and B. D. Woerner, "Spatial fading correlation function of circular antenna arrays with Laplacian energy distribution," vol. 6, no. 5, pp. 178-180, May 2002.

[6] P. D. Teal, T. D. Abhayapala, and R. A. Kennedy, "Spatial correlation for general distributions of scatterers," IEEE Signal Process. Lett., vol. 9, no. 10 , pp. 305-308, Oct. 2002.

[7] K. Mammasis and R. W. Stewart, "Spherical statistics and spatial correlation for multielement antenna systems," EURASIP J. Wirel. Commun., vol. 2010, Dec. 2010.

[8] J. H. Lee and C. C. Cheng, "Spatial correlation of multiple antenna arrays in wireless communication systems," Progress in Electromagnetics Research, vol. 132, pp. 347-368, 2012.

[9] R. A. Kennedy, Z. Khalid, and Y. F. Alem, "Spatial correlation from multipath with 3D power distributions having rotational symmetry," in Proc. Int. Conf. Signal Processing and Communication Systems (ICSPCS), Gold Coast, Australia, Dec. 2013, p. 7.

[10] J. A. Tsai and B. D. Woerner, "The fading correlation function of a circular antenna array in mobile radio environment," in Proc. Global Telecommunications Conference (GLOBECOM), vol. 5, 2001, pp. 32323236.

[11] R. M. Buehrer, "The impact of angular energy distribution on spatial correlation," in Proc. Vehicular Technology Conference (VTC), vol. 2, 2002, pp. 1173-1177.

[12] S. K. Yong and J. S. Thompson, "A three-dimensional spatial fading correlation model for uniform rectangular arrays," IEEE Antennas Wireless Propag. Lett., vol. 2, no. 1, pp. 182-185, 2003.

[13] J. Zhou, S. Sasaki, S. Muramatsu, H. Kikuchi, and Y. Onozato, "Spatial correlation for a circular antenna array and its applications in wireless communication," in Proc. Global Telecommunications Conference (GLOBECOM), vol. 2, 2003, pp. 1108-1113.

[14] J. S. K. Raj, S. P. Arokiasamy, N. Vikram, and J. Schoebel, "Spatial correlation and MIMO capacity of uniform rectangular dipole arrays," IEEE Antennas Wireless Propag. Lett., vol. 7, pp. 97-100, 2008.

[15] R. A. Kennedy and P. Sadeghi, Hilbert Space Methods in Signal Processing. Cambridge, UK: Cambridge University Press, Mar. 2013.

[16] J. B. Andersen and K. I. Pedersen, "Angle-of-arrival statistics for low resolution antennas," IEEE Trans. Antennas Propag., vol. 50, no. 3, pp. 391-395, Mar. 2002.

[17] H. M. Jones, R. A. Kennedy, and T. D. Abhayapala, "On dimensionality of multipath fields: Spatial extent and richness," in Proc. IEEE Int. Conf. Acoustics, Speech, and Signal Processing, ICASSP'2002, vol. 3, Orlando, FL, May 2002, pp. 2837-2840.

[18] S. Trapani and J. Navaza, "AMoRe: Classical and modern," Acta Crystallogr. Sect. D, vol. 64, no. 1, pp. 11-16, Jan. 2008.

[19] D. Colton and R. Kress, Inverse Acoustic and Electromagnetic Scattering Theory, 3rd ed. New York, NY: Springer, 2013.

[20] T. Risbo, "Fourier transform summation of Legendre series and Dfunctions," J. Geodesy, vol. 70, no. 7, pp. 383-396, 1996. 